\title{
Hemoglobina Köln diagnosticada em programa de triagem neonatal em São José do Rio Preto, SP
}

\author{
Emanuele C. Schiaveto ${ }^{1}$ \\ Alessandra Vidotto 1 \\ Fátima A.M. Siqueira ${ }^{1}$ \\ Paulo C. Naoum \\ Agnes C. Fett-Conte \\ Claudia R. Bonini-Domingos ${ }^{1}$
}

\begin{abstract}
Alteraçôes genéticas em que a mutação de aminoácidos nas globinas afeta a estrutura da molécula tornando-a instável são classificadas como hemoglobinas instáveis. Devido à grande diversidade dos pontos de mutaçôes por substituições e deleçôes de aminoácidos, as formas de instabilização se apresentam muito variadas. A hemoglobina Köln é a variante instável descrita com maior freqüência na literatura e a terceira descoberta no Brasil, as outras são Hb Niterói e Hb Hasharon. Anemia moderada, ictericia e presença de urina escura caracterizam as manifestações clínicas da Hb Köln. Em programa de triagem neonatal identificamos uma criança com suspeita de heterozigose para hemoglobina Köln, confirmada por procedimentos eletroforéticos e HPLC. Avaliações por diferentes metodologias laboratoriais e estudo familiar auxiliam no diagnóstico precoce, possibilitando minimizar os sintomas decorrentes da hemoglobina anormal e a realização do aconselhamento genético e educacional destas alteraçôes hereditârias.

Rev.bras.hematol.hemoter.,2002,24(1): 41-44
\end{abstract}

Palavras-chaves: Hemoglobinas instáveis, hemoglobina Köln, triagem neonatal

\section{Introdução}

As hemoglobinas instáveis constituem um grupo de alterações genéticas em que a mutação de aminoácidos nas globinas alfa ou beta afeta a estrutura da molécula, tornando-a instável. A avaliação da estabilidade da molécula de hemoglobina é realizada por métodos físicoquímicos bem definidos atualmente, entre os quais destacam-se o aquecimento da solução de hemolisado entre 50 e $60^{\circ} \mathrm{C}$, a incubação com isopropanol $(\mathrm{HCl})$ a $37^{\circ} \mathrm{C}$, ou a agitação vigorosa do tubo contendo o hemolisado. Assim, as hemoglobinas que precipitam mais rapidamente do que as normais são designadas instáveis (1).

Devido à grande diversidade dos pontos de mutações por substituições e deleções de aminoácidos, as formas de instabilização se apresentam muito variadas. Algumas hemoglobinas são discretamente instáveis e não estão associadas com sintomas clínicos, enquanto outras se precipitam com grande intensidade, causando anemias hemolíticas. Essas anemias podem ser graves, com acentuada diminuição dos níveis de hemoglobina e reticulocitose, ou então, podem apresentar-se com discreto quadro hemolítico acompanhado

1 - Laboratórios de Hemoglobinas e Genética das Doenças Hematológicas, Departamento de Biologia, IBILCE, UNESP, São José do Rio Preto, SP

2 - Departamento de Biologia Molecular, FAMERP, São José do Rio Preto, SP

Correspondência para: C.R. Bonini-Domingos

IBILCE-UNESP, Departamento de Biologia, Laboratórios de Hemoglobinas e Genética das Doenças Hematológicas. Rua Cristóvão Colombo, 2265. 15054-000. São José do Rio Preto. SP. Brasil.

Fax: 55 (17) 221-2390. E-mail: bonini@bio.ibilce.unesp.br 
por modesta reticulocitose. Uma das principais características das hemoglobinas instáveis que causam anemia hemolítica é a presença de corpos de Heinz (2).

A hemoglobina Köln é a variante instável com maior freqüência na literatura e a terceira descoberta no Brasil, as outras são Hb Niterói e $\mathrm{Hb}$ Hasharon (3). É resultante da substituição do aminoácido valina por metionina na posição 98(FG5) GTG $\rightarrow \underline{\text { ATG }}$ da cadeia beta globina. A hemoglobina anormal varia de 10 a 15\% e apresenta um aumento na afinidade ao oxigênio. É originária de europeus, afro-americanos e japoneses $(4,5,6,7)$. Anemia moderada, icterícia e presença de urina escura caracterizam as manifestações clínicas da $\mathrm{Hb} \operatorname{Köln}(7,8,9)$. Intervenções como a esplenectomia diminui a anemia e melhora o quadro clínico causado pela doença $(10,11)$.

\section{Descrição do caso}

Para triagem de hemoglobinopatias em neonatos utilizamos sangue de cordão umbilical colhido em tubos contendo EDTA $5 \%$, por gravidade e não por ordenha do cordão, identificados como RN de (nome da mãe e número do prontuário hospitalar da mãe) e acondicionadas em geladeira até o momento das análises, não excedendo cinco dias. As coletas foram realizadas no Hospital de Base da Faculdade de Medicina de São José do Rio Preto, SP, e o termo de consentimento devidamente preenchido e assinado. Após os procedimentos de identificação, as amostras foram submetidas aos testes de triagem como: resistência globular osmótica em $\mathrm{NaCl}$ a 0,36\% (12), morfologia eritrocitária (2), eletroforese em acetato de celulose $\mathrm{pH}$ alcalino (13), os quais permitem a identificação de hemoglobinas normais e anormais nesta faixa etária.

No presente caso observou-se negatividade no teste de resistência globular osmótica em $\mathrm{NaCl}$ a $0,36 \%$ e a morfologia eritrocitária apresentou-se com discretas alterações. $\mathrm{Na}$ eletroforese em acetato de celulose $\mathrm{pH}$ alcalino identificou-se a presença de banda migrando entre as hemoglobinas $\mathrm{A}_{2}$ e $\mathrm{S}$, quando $\mathrm{O}$ hemolisado era realizado com saponina a $1 \%$ (14). No procedimento eletroforético realizado com hemolisado preparado com clorofórmio (14), observamos o desaparecimento desta banda anômala, sugerindo a instabilidade da molécula. Os perfis eletroforéticos, nas preparações com saponina e clorofórmio, foram realizados utilizando-se gel de agarose para hemoglobina alcalina da CELM. Em eletroforese pH ácido (15), o padrão de migração foi semelhante à $\mathrm{Hb} \mathrm{A}$.

Diante da suspeita de hemoglobina instável foram realizados os procedimentos laboratoriais padrão para caracterização destas formas anormais de hemoglobina e a amostra apresentou positividade aos testes de instabilidade térmica (16) e de precipitação pelo isopropanol (17) e presença de corpos de Heinz (18). A banda anormal específica foi confirmada por focalização isoelétrica em gel de agarose (14). A eletroforese de cadeias globínicas (14) apresentou padrão de migração das globinas, compatível com a suspeita de Hb Köln.

A criança e seus familiares foram chamados para aconselhamento genético educacional e a presença da fração anormal foi confirmada em heterozigose na criança e sua mãe, por Cromatografia Líquida de Alta Resolução (HPLC) utilizando equipamento Variant da Bio-Rad e kit de diagnóstico para talassemia beta heterozigota. A fração anormal na criança apresentou 19,2\% e tempo de retenção de 1.58. Na mãe observou-se o mesmo tempo de retenção e quantidade de $17,8 \%$ da fração anormal.

\section{Discussão}

A relação entre o aminoácido substituído (valina por metionina) e a instabilidade da hemoglobina Köln sugere que a valina está em contato com o heme na cadeia beta globina. A substituição da valina por metionina na posição FG5 causa mudança na configuração da globina beta e alterações na redução enzimática do ferro, resultando no aumento 
da conversão de metahemoglobina em hemoglobina desnaturada $(7,19)$.

Quando a Hb Köln está na conformação oxigenada, a molécula está saturada e é estável. A transição para o estado desoxigenado resulta na perda do grupo heme, instabilidade, precipitação e formação de corpos de Heinz, que são produtos da degradação citoplasmática afetando a membrana celular $(7,20)$. Esta substituição de aminoácido causa instabilidade intermediária da molécula e o paciente sofre processo hemolítico crônico de moderado a severo e esplenomegalia $(21,22)$. Na Hb Köln, a afinidade ao oxigênio é aproximadamente 2,5 vezes maior que a $\mathrm{Hb} \mathrm{A}$ e o efeito Bohr é normal (23).

Como o indivíduo portador pode apresentar sintomas de anemia hemolítica desde o nascimento, devido à instabilidade da molécula, enfatizamos a necessidade de análises laboratoriais criteriosas nos estudos de hemoglobinopatias em neonatos. Estes procedimentos auxiliam no diagnóstico precoce destas alterações, possibilitando minimizar os sintomas decorrentes da hemoglobina anormal, e auxiliam na realização do aconselhamento genético e educacional destas alterações hereditárias.

\section{Köln Hemoglobin found in Neonatal Screening Program in São José do Rio Preto, São Paulo, Brazil}

Emanuele C. Schiaveto, Alessandra Vidotto, Fátima A.M. Siqueira, Paulo C. Naoum, Agnes C. Fett-Conte, Claudia R. Bonini-Domingos

\section{Abstract}

Hemoglobinopathies are a diverse group of inherited recessive disorders that include thalassemias and sickle cell disease. They were the first genetic diseases to be characterized at the molecular level and consequently have been used repeatedly as a prototype for the development of new techniques of mutation detection. A major group of the structural bemoglobins exhibit the property of instability in solution, resulting from their altered molecular structures. Mutation of amino acids in the globin affects the structure of the molecule turning it unstable and they are classified as unstable bemoglobins. Due to the great diversity of the mutation points, substitutions and deletions of amino acids, the unstable forms are very varied. Hemoglobin Köln is the unstable variant most described in literature and the third discovered in Brazil, the others are $\mathrm{Hb}$ Niteró $i$ and $\mathrm{Hb}$ Hasharon. Moderate anemia, jaundice and the presence of dark urine characterize Hb Köln's clinical manifestations. In a neonatal screening program we identified a child suspected of having hemoglobin Köln, which was confirmed by electrophoretic procedures and HPLC. Evaluations by different laboratory examinations and the family study aided in a precocious diagnosis, thereby facilitating to minimize the current symptoms of the abnormal bemoglobin, and thus the family received support with genetic and educational counseling of these bereditary alterations.

Rev.bras.hematol.hemoter.,2002,24(1): 41-44

Keywords: Unstable hemoglobins, Köln hemoglobin, neonatal screening

\section{Referências Bibliográficas}

1. Naoum PC. Hemoglobinopatias $e$ talassemias. Sarvier. São Paulo, 1997.

2. Bonini-Domingos CR. Hemoglobinopatias no Brasil: variabilidade genética e metodologia laboratorial. São José do Rio Preto, 1993. Tese (Doutoramento em Ciências Biológicas) - Instituto de Biociências Letras e Ciências Exatas, Universidade Estadual Paulista.

3. Naoum PC, Domingos CRB, Moreira HW \& Alvares FoF. $\mathrm{Hb} \mathrm{Köln} \mathrm{or} \alpha_{2} \beta_{2} 98$ (FG5) ValMet in Brazil. Simpósio Nacional de Hemoglobiopatias. São José do Rio Preto, SP, Brasil, 1993.

4. Miller DR, Weed RI, Stamatoyannopoulos G \& Yoshida A. Hemoglobin Köln disease occurring as a fresh mutation: erythrocyte metabolism and survival. Blood. 1971; 38:715-729.

5. Pedersen PR, McCurdy PR, Wrightstone RN, 
Wilson JB, Smith LL \& Huisman THJ. Hemoglobin Köln in a black: pre- and postsplenectomy red cell survinal (DF32P and $51 \mathrm{Cr}$ ) and the pathogenesis of hemoglobin instability. Blood. 1973; 42:771-781.

6. Carrel RW, Lehmann $\mathrm{H}$ and Hutchison HE. Haemoglobin Köln ( $\beta 98$ Valine-Metionine): an unstable protein causing inclusion body anaemia. Nature. 1966; 210: 2915-916.

7. Miranda SRP, Fonseca SF, Figueiredo MS, Yamamoto M, Grotto HZW, Saad STO and Costa FF. Hb Köln [ $\alpha_{2} \beta_{2} 98$ (8FG5) Val-Met] identified by DNA analysis in a Brazilian family. Brazilian Journal of Genetics. 1997; 20:745-748.

8. Fairbanks VF, Opfell RW and Burgert Jr.EO. Three families with unstable bemoglobinopathies (Köln, Olmsted and Santa Ana) causing hemolytuc anemia with inclusion bodies and pigmenturia. Am. J. Med. 1969; 46: 344-359.

9. Lolskin GB and Miller DR. Heme synthesis in bereditary hemolytic anemias: descrease of $\delta$-aminolevulinic synthetase in hemoglobin Köln disease. Pediat. Res. 1976; 10:702-706.

10. Scott JL, Fritsch EF and Maniatis T. Molecular Cloning. Cold spring Harbor Laboratories, New York, 1989.

11. Vaughan Jones R, Grimes AJ, Carrel RW and Lehman H. Köln haemoglobinopathy: further data and a comparison with other hereditary Heinz bodies anaemias. Brit. J. Haematol. 1967; 13:394-408.

12. Silvestroni E, Bianco I. Screening for microcytemiain Italy: analysis of data collected in the past 30 years. Am. J. Hum. Genet 1975; 27:198-212.

13. Marengo-Rowe AJ. Rapid eletrophoresis and quantitation of haenoglobin on cellulose acetate. J. Clin. Path. 1965; 18:790-792.

14. Naoum PC. Eletroforese, técnicas e diagnóstico. São Paulo: Santos, 1998.

15. Vella F. Acid agar gel eletrophoresis of buman hemoglobin. Am. J. Clin. Path. 1968; 49: 440-442.

16. Dacie JV, Lewis SM. "In: Practical Haematology" 6th ed. Churchill, London, p.516, 1985.

17. Carrel RW, Kay R. A simple method for detection of unstable hemoglobins. British J. of Hematol. 1972; 23:615-619.

18. Papayannopoulos R, Stamatoyannopoulos G. Stains for inclusions bodies. "In: Standartization of laboratory reagents and methodos for detection of haemoglobinopathies". Atlanta: Hew publications, 1974.

19. Harley JD and Mauer AM. Studies on the formation of Heinz bodies. II. The nature and significance of Heinz bodies. Blood. 1961; 17:418-433.

20. Eisenger J, Flores J, Tyson JA and Shohet SB. Fluorescent cytoplasm and Heinz bodies of hemoglobin Köln erytrocytes: evidence for intracellular heme catabolism. Blood. 1985; 65: 886-893.

21. Honig GR, Adams III JG. Human bemoglobin genetics. Springer Verlag. Wien., 1986.

22. Lehmann $\mathrm{H}$ and Huntsman RG. Man's haemoglobins. North-Holland publishing company - Amsterdam. Oxford, 1974.

23. Schmidt RM. Abnormal Haemoglobins and Thalassaemia. London, Academic Press, 1975.

Recebido 13/08/01

Aceito: 26/01/02 